\title{
GNINA 1.0: Molecular docking with deep learning
}

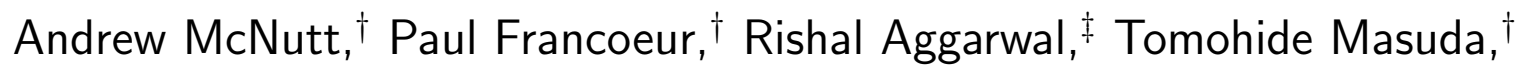 \\ Rocco Meli, ${ }^{\llbracket}$ Matthew Ragoza, ${ }^{\dagger}$ Jocelyn Sunseri, ${ }^{\dagger}$ and David Ryan Koes ${ }^{*} \dagger$ \\ $\dagger$ Department of Computational and Systems Biology, University of Pittsburgh, Pittsburgh, \\ $P A$ \\ $\ddagger$ Center for Computational Natural Sciences and Bioinformatics, International Institute of \\ Information Technology, Hyderabad 500 032, India \\ \Department of Biochemistry, University of Oxford, Oxford, United Kingdom \\ E-mail: dkoes@pitt.edu
}

\begin{abstract}
Molecular docking computationally predicts the conformation of a small molecule when binding to a receptor. Scoring functions are a vital piece of any molecular docking pipeline as they determine the fitness of sampled poses. Here we describe and evaluate the 1.0 release of the GNINA docking software, which utilizes an ensemble of convolutional neural networks (CNNs) as a scoring function. We also explore an array of parameter values for GNINA 1.0 to optimize docking performance and computational cost. Docking performance, as evaluated by the percentage of targets where the top pose is better than $2 \AA$ root mean square deviation (Top1), is compared to AutoDock Vina scoring when utilizing explicitly defined binding pockets or whole protein docking. GnINA, utilizing a CNN scoring function to rescore the output poses, outperforms AutoDock Vina scoring on redocking and cross-docking tasks when the
\end{abstract}


binding pocket is defined (Top1 increases from $58 \%$ to $73 \%$ and from $27 \%$ to $37 \%$, respectively) and when the whole protein defines the binding pocket (Top1 increases from $31 \%$ to $38 \%$ and from $12 \%$ to $16 \%$, respectively). The derived ensemble of CNNs generalizes to unseen proteins and ligands and produces scores that correlate well with the root mean square deviation to the known binding pose. We provide the 1.0 version of GNINA under and open source license for use as a molecular docking tool at https://github.com/gnina/gnina.

\section{Introduction}

Molecular docking is a computational procedure in which the non-covalent bonding of molecules, e.g. a protein receptor and a ligand, is predicted. This prediction outputs the conformation and, usually, the binding affinity of the small molecule in its predicted minimal energy state and is used to virtually screen large libraries of compounds. ${ }^{1-3}$ Docking is composed of two main steps: sampling and scoring. Sampling refers to an extensive search of the conformational space of the molecules being docked. This conformational space is vast, due in part to both the receptor and ligand being flexible allowing for each molecule to adjust its shape due to the influence of the other. In order to constrain this large conformational space, the receptor is typically kept rigid. The other vital piece of molecular docking is the scoring function. Every sampled pose is evaluated by the scoring function for its fitness. The fitness determines the conformations that are retained from sampling and is used to rank the retained poses in the order of their likelihood of being correct. The final output of docking is a set of ranked poses of the docked molecule.

Determination of the correct binding pose of a small molecule is a prerequisite for determining its binding affinity and affords the opportunity to utilize the pose for lead optimization. Correct evaluation of binding affinity is critical for downstream tasks such as virtual screening or for determining if a compound is important for more experimental analysis. Molecular docking must compute a pose and a binding affinity quickly for it to beneficial 
when millions of ligands are being queried in a drug discovery pipeline. 1 Sampling the entire conformational space of a molecule is computationally demanding; therefore, we compromise on the speed and accuracy of docking to provide poses that are close to native while not requiring the full search of conformational space. This compromise requires docking software to focus on the accuracy and ranking power of the scoring function to highlight low energy conformations and reduce extraneous sampling.

Scoring functions provide a mapping from the conformational space of the ligand and receptor to the set of real numbers so that poses may be ranked. Typically, scoring functions are grouped into three categories; knowledge based, physics based, and empirical.1 Knowledge based scoring functions leverage the statistics from a set of structural binding data. A number of geometric properties are computed from structures of protein-ligand complexes, such as atom-atom pairwise contacts. The calculated frequencies can be used in a method such as potential of mean force (PMF), which creates a potential based on the Boltzmann

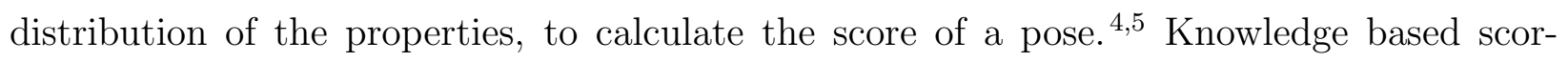
ing functions can be biased by features present in their training sets though calculations of scores are quick at test time. 1 They require a large database of known structures and can be difficult to interpret when trying to understand a score. ${ }^{[6}$ Physics based scoring functions, often referred to as force fields, utilize physically derived energetics of interactions to compute scores. The final score is a summation of energy terms such as Coulombic and Van der Waals forces. ${ }^{7}$ Accuracy of physics based scoring functions are limited by their complexity and the assumptions we place on the fundamental forces dictating interactions between molecules, though understanding of these forces is continually increasing. $\underline{8}$

Empirical scoring functions address the limitations of physics based scoring functions by using a combination of manually selected energy terms. Rather than giving each energy term identical weighting, the weights of each term are determined via a fit to experimental data. A large proportion of docking software use empirical scoring functions, including X-Score, AutoDock Vina, and ChemScore.11 Unlike knowledge based scoring functions, 
empirical and physics based scoring functions may be easily interpreted to determine the contributing factors of a given score since each energy term can be individually queried. Fitting empirical scoring function requires a plethora of experimental structural data and prevents the combination of terms from separately trained scoring functions. The three categories of scoring functions are limited to features extracted from structural information and often assume there is a linear relationship between the features and the binding affinity. AutoDock Vina (called "Vina") utilizes an empirical scoring function explicitly tuned to structural data. ${ }^{10}$ The Vina scoring function is a weighted sum of atomic interactions. Steric, hydrophobic, and hydrogen bonding interactions are calculated and are adjusted by the number of rotatable bonds to account for entropic penalties. The weights of the terms were computed via a non-linear fit to structural data. Nguyen et al. 12] show that Vina can more accurately predict the binding pose than its predecessor, AutoDock $4 . \frac{13}{}$ Vina demonstrates the power of modelling non-linear relationships with its increased docking performance. Therefore, we search for alternative scoring functions that are able to model non-linear relationships between inputs.

Machine learning (ML) represents another growing class of scoring functions. ${ }^{8}$ ML algorithms learn arbitrary relationships between observations and outputs while classical scoring functions assume a specific functional form. ${ }^{114}$ There has been considerable progress in other biomedical fields with the utilization of ML models. ${ }^{15}$ However, machine learning algorithms require a large amount of data to properly generalize to unseen information. The last 20 years has seen a noteworthy increase in the quantity of available protein structures. $\frac{16}{}$ A plethora of databases annotate structural data with experimental binding affinity data, including PDBbind and BindingDB. $\frac{17-19}{19}$ This information has been utilized to leverage machine learning algorithms as scoring functions. A number of traditional ML approaches have been used as scoring functions, including random forests (RF-Score ${ }^{\sqrt{20}}$ and SFCScore ${ }^{21}$ ), support vector machines (SVR-Score, ${ }^{22}$ ID-Score, ${ }^{23}$ SVR-EP ${ }^{24}$ ), artificial neural networks (NNscore ${ }^{25}$ and BsN-Score ${ }^{26}$ ), and gradient boosted decision trees (BT-dock ${ }^{[27}$ and ESPH T-Bind ${ }^{28}$ ). 
These ML methods have been able to match or exceed existing traditional scoring functions. ML methods allow a more robust fit to training data, but are limited to features manually extracted from structural data.

Deep learning (DL) methods allow direct inference of features from inputs. They learn a representation of the inputs via layers of simple, non-linear models which transform the representation to higher abstractions to learn complex functions. ${ }^{29}$ DL methods have demonstrated success in a variety of fields, such as computer vision and natural language processing. ${ }^{30 \mid 31}$ In recent years, there has been significant progress with DL methods in the drug discovery field with many models employing a convolutional framework. Convolutional neural networks (CNN) leverage convolutions to infer features directly from input tensors, usually

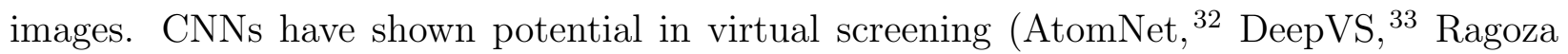

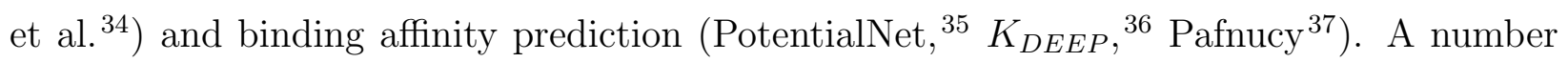
of methods have been proposed to capitalize on the power of DL scoring functions. MedusaNet uses a CNN within the docking pipeline to guide the sampling of the base docking method. $\frac{38}{38}$ The base docking method, Medusa, provides a variety of ligand poses. The CNN evaluates the $3 \mathrm{D}$ coordinate representation of the poses to determine if a pose should be retained. Nguyen et al. $\frac{[39}{}$ describe a generative adversarial network (GAN) for pose prediction. Their network utilizes an encoder with low-dimensional mathematical representations of the protein-ligand complex and a decoder utilizing convolutional layers to generate and rank ligand poses for the D3R grand challenge. Masuda et al. $\stackrel{40}{4}$ use a receptor structure as the prior to their GAN to sample novel ligands appropriate to the identified binding site.

Previous work has largely evaluated deep learning protein-ligand scoring on already generated poses. In this work, we describe and comprehensively evaluate version 1.0 of the GNinA molecular docking software, a fork of SMINA ${ }^{41}$ and AutoDock Vina ${ }^{10}$ that supports CNN scoring as an integral part of the docking workflow. GNINA is evaluated here for its ability to properly score and rank binding poses for protein-ligand complexes. We describe how the default settings which balance docking accuracy and runtime were determined, including 
the selection of a default ensemble of CNN models. Performance of GNinA is evaluated for the redocking, cross-docking, flexible docking, and whole protein docking tasks and is found to significantly outperform SminA/Vina in all cases.

\section{Methods}

The docking pipeline of GNINA (Figure 1) is described in detail, providing background for the derivation of default usage. A default CNN ensemble is selected for optimizing the docking performance and runtime of the docking pipeline. This ensemble is then used to investigate the different CNN scoring options available to the user, followed by a thorough investigation of the docking parameters. Finally, we examine both the generalizability and scoring power of GNINA.
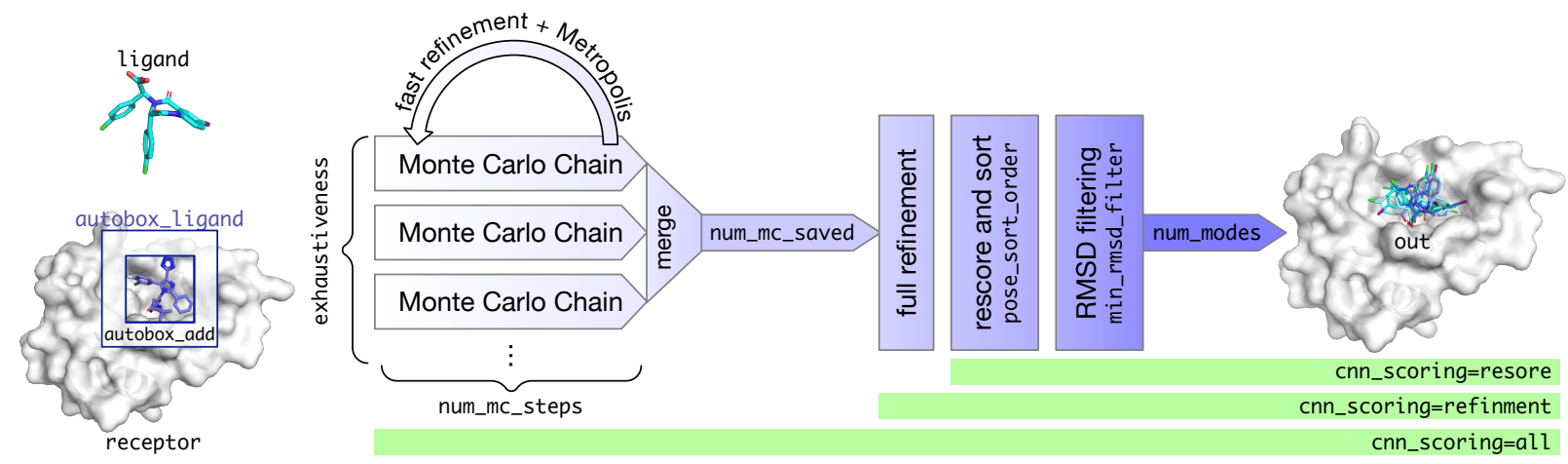

Figure 1: The GNINA sampling and scoring algorithm shown with relevant commandline parameters and the scope of CNN scoring.

\section{Molecular Docking Pipeline}

GNinA is a fork of SMInA ${ }^{41}$ which is a fork of Vina. ${ }^{10}$ The docking pipeline of GNINA utilizes the enhanced support for scoring enabled in SMINA to allow the use of CNNs as scoring functions. In typical usage, GNINA is provided with a receptor structure, a ligand structure, and a specification for a binding site on the receptor.

Open Babel, $\stackrel{42 / 43}{a}$ a chemical toolbox allowing the reading and writing of over 100 chemical 
gnina --receptor 1BCU_PROT.pdb --ligand 1BCU_LIG.sdf --out

$1 \mathrm{BCU}_{-}$gnina_poses.sdf.gz --autobox_ligand 1BCU_LIG.sdf

--autobox_add 4 --cnn crossdock_default2018 dense_3

--cnn_scoring rescore --exhaustiveness 8 --num_mc_saved 50

--cnn_rotation 0 --num_modes 9 --min_rmsd_filter 1

\section{Example Gnina Usage}

file formats, is used for parsing the inputs, allowing commonly used structural data formats (e.g. PDB, sdf, mol, etc.) as well as gzipped versions of such files to be used. The binding site can be specified as a Cartesian box or by providing a ligand file (autobox_ligand). When autobox_ligand is used to define the binding site, a rectangular prism is constructed using the minimum and maximum values for the $x, y$, and $z$ coordinates of the ligand to which additional spacing (autobox_add) is added in every dimension (Figure 1). In GNiNA, if any side of this auto-generated box is smaller than the longest distance between any two atoms in the ligand, then those sides are extended to that longest distance, ensuring that the ligand can rotate freely within the defined box without incurring an out-of-box penalty that is applied to all docked poses to constrain them to the specified binding site search space.

Next, the scoring functions are setup. Similar to Smina, if the user opts to not use the CNN scoring function for a part of the pipeline they can specify their own empirical scoring function to GNina or choose one of the built-in scoring functions, i.e. Vina, Vinardo, 44 etc. CNN scoring functions can be specified by providing model and/or weights files or by selecting a built-in model. The available built-in CNN models include crossdock_default2018, dense, general_default2018, redock_default2018, and default2017, each of which is trained using different training data and/or a different model architecture. Additionally, each model, except for default2017, has five variants that are trained on the same data and have the same architecture, but are initialized with a different random seed. For each CNN model type, we refer to the variant with the highest docking performance as the base model name, and the remaining variants are given sequential numbers (i.e. general_default2018, general_default2018_1, general_default2018_2, general_default2018_3, and general_ 
default2018_4); the ensemble of these five variants is denoted with '_ensemble' (i.e. general_ default2018_ensemble). The architecture and training of these models are described elsewhere. ${ }^{34145} \mathrm{CNN}$ calculations are performed using the cuDNN accelerated Caffe deep learning framework. $\stackrel{46}{4}$ These models are trained to predict both a pose score (a probability that the pose has a low root mean square deviation (RMSD) to the binding pose) and the binding affinity (pK). The pose score is used for all pose optimization tasks. The scoring function used in each step of the GNINA pipeline is determined by cnn_scoring (defined below), defaulting to only using the CNN scoring for the final resorting of ligand conformations and the empirical scoring function everywhere else in the pipeline.

The docking procedure uses Monte Carlo sampling to search the ligand conformational space. exhaustiveness (default 8) defines the number of Monte Carlo chains that are run for the ligand. Chains are run in parallel using up to cpu threads. The number of steps for the Monte Carlo chains are calculated based on the number of mobile atoms and the number of degrees of freedom within the ligand. This calculation can be ignored and the user may explicitly specify the number of steps for the Monte Carlo chains with num_mc_steps. Each step of a Monte Carlo chain mutates the ligand by randomly selecting one of the following operations: random translation, random rotation of the entire molecule, or randomly setting the torsional angles of the ligand. The Monte Carlo process selects random resetting of the torsional angles with a higher probability than the other mutations. After the mutation, an approximate energy minimization of the ligand is performed. If an empirical scoring function is specified for guiding sampling, this minimization is performed using a fast, grid-based approximation of that scoring function. A grid is pre-calculated for each ligand atom type and a single atom of that type is used to calculate values for each grid point. The full ligand is scored by interpolating values from the grid for each of its atoms and summing the result. If a CNN scoring function is specified, no such approximation is used since CNN scoring is not additive with respect to the individual atoms. $\frac{47}{4}$ The score for the minimized conformation determines if it will be accepted using the Metropolis acceptance criterion. Each Monte 
Carlo chain retains its top scoring ligand conformations, and the number retained is user configurable with num_mc_saved (default 50).

Following the completion of Monte Carlo sampling, the saved conformations from each Monte Carlo chain are aggregated and the num_mc_saved top scoring conformations are retained for further analysis. The top scoring conformations are then refined. If an empirical scoring function is specified for refinement (cnn_scoring set to "none" or "rescore"), a functional formulation, rather than a grid approximation, of the scoring function is used to carry out refinement in order to get the locally optimal pose. Refinement shifts the ligand pose to a local energy minimum using the gradients of the scoring function. After the ligand pose has been refined, the final affinities and scores are calculated for the pose using the specified CNN models and/or the specified scoring function. Finally, the top scoring conformations are sorted by pose_sort_order (default "CNNscore") and output with Open Babel in the user-specified format if an output file was provided.

The usage of the CNN models within the docking pipeline can be selected by the user using cnn_scoring (Figure 1). If "none" is selected for cnn_scoring, then the CNN models are not used at all in the docking pipeline, making the pipeline essentially identical to the Smina pipeline. The only differences are that Smina computes with double (64 bit) precision rather than the single (32 bit) precision used by GNINA and Smina does not include the autobox_extend parameter when creating the sampling box. When "rescoring" is selected (the default), the specified CNN models are not used until the final sorting of resultant ligand conformations after their refinement with the empirical scoring function. In this case, the specified CNN models are used to score each of the ligand conformations and the output ligand conformations are resorted based on the score calculated by the CNN model(s). The "refinement" option utilizes the CNN for the refinement of the ligand poses after they have been selected by the Monte Carlo chains and then sorts the refined ligand conformations by the CNN score for output. Finally, the "all" option utilizes the CNN for all aspects of the docking pipeline including the minimization within the Monte Carlo chains, the refinement 
after the Monte Carlo chains, and the sorting of the final output.

\section{Flexible Docking}

Molecular docking is often performed using a rigid protein target and only samples the conformational space of the ligand, as described above. This is a good approximation when redocking to a receptor that does not undergo conformational change upon ligand binding, but it fails to accurately represent the biochemical aspects of the system when the protein undergoes significant structural changes upon binding. ${ }^{48}$ Allowing the whole target to be flexible is too computationally expensive for docking; however, some protein flexibility can be accounted for by sampling the conformational space of side chains in the binding site $\mathrm{e}^{49}$ while leaving the backbone fixed. The local flexibility of the binding site could be especially beneficial for cross-docking, i.e. docking a ligand to a non-cognate receptor. Assuming a rigid receptor during cross-docking is not realistic when potentially very different ligands are docked to the same receptor.

GnINA allows the sampling of side chain, but not backbone, conformational space. More specifically, side chain torsion angles are sampled as part of the Monte Carlo search. Side chain flexibility can be specified manually or semi-automatically in several different ways:

- flexible side chains can be defined in a PDBQT file (flex parameter),

- they can be selected using a comma-separated list of residue identifiers (chain, residue number, and, optionally, insertion code; flexres parameter),

- or they can be selected based on their distance from a given ligand (flexdist and flexdist_ligand parameters).

The last option, used in this work, is similar to the autobox_ligand option described above. The flexdist parameter allows the specification of a threshold distance from the flexdist_ligand. If a residue has any side chain atoms that are within this distance of the specified ligand, then the entire residue side chain is marked as flexible. 
Given the increased computational cost of sampling side chain conformations, two additional options are provided for flexible docking. flex_limit allows users to specify a hard upper bound for the number of flexible side chains. If flex_limit is exceeded for a particular system, docking does not run and GninA terminates with a warning. This option is particularly useful to avoid possible bottlenecks in large virtual screening tasks. The less strict flex_max option allows users to prune the list of flexible side chains in order to make the calculation more manageable for large systems; if there are more than flex_max side chains identified as flexible, only the conformational space of the flex_max closest to flexdist_ligand is sampled during docking.

Flexible side chains are selected at the very beginning of the docking procedure and the selection is not updated during sampling. When using autobox_ligand to automatically define the search space as described above, flexible side chains are included in the calculation of the box bounds. The usage of CNN models for docking with flexible side chains can be selected by the user in the same way it is done for docking with a rigid receptor, as described above.

\section{Data}

There are two primary ways to evaluate molecular docking: redocking a cognate ligand to its receptor and docking a ligand to a non-cognate receptor (cross-docking). In order to best evaluate the performance of GNINA for molecular docking, we evaluate its performance on both of these tasks. Redocking the cognate ligand demonstrates the sampling and scoring power of the molecular docking pipeline, as the RMSD from the crystal pose can be measured to exactly determine the accuracy of the produced poses. Analysis of redocking requires a set of high quality structures in which the native binding pose of the ligand has been solved. For this purpose we utilize the PDBbind refined set v.2019.18 The PDBbind database is a curated set of protein-ligand complexes containing both structural information and binding affinity. The PDBbind database is updated annually with new experimentally determined 
structures annotated with binding affinity data. The refined set is a subset of the entire PDBbind dataset that retains only the structures with resolution higher than $2.5 \AA$, high quality affinity measurements, and binary protein-ligand complexes. The 2019 release of the refined set contains 4,852 high quality crystal structures of native protein-ligand binding poses.

However, redocking is not the normal use case of a molecular docking pipeline. Docking will ordinarily be performed on proteins-ligand pairs that have no co-crystallized structure. Often a new ligand will be docked into a receptor whose co-crystallized ligand is a different molecule. Wierbowski et al. $\stackrel{50}{ }$ recently published a dataset that provides a benchmark precisely for this task. This cross-docking dataset provides a meaningful method for the evaluation of the ligand RMSD from the known and predicted poses. A reference structure is selected for the protein, then the "known" binding pose of a ligand is defined by the ligand's position when an alignment is performed between the reference structure and the ligand's co-crystal receptor structure. The dataset is composed of 94 unique protein binding pockets and 4,399 unique ligands, with an average of 46 ligands per target.

Both of the datasets were filtered to ensure the protein-ligand structures can be parsed by GninA. ProDy $\sqrt{51}$ was used to separate the complexes into protein and ligand files while removing any water or other extra crystallized molecules. Our goal is the binding pose prediction of a small molecule at its target site, therefore we utilized RDKit ${ }^{52}$ to filter both the redocking and cross-docking datasets to include only ligands with molecular masses greater than 150 Da and less than 1000 Da. Any ligand that was not able to be parsed with RDKit was also removed. In addition, we visually inspected the ligands of the cross-docking dataset with Pymol ${ }^{[53}$ to ensure that each ligand for a given binding pocket overlapped. If a ligand was non-overlapping it was marked as problematic. Lastly, for the UROK pocket, $4 \mathrm{ZKO}$ was removed as the receptor did not align properly and the ligands from $4 \mathrm{MNW}$ and 4MNX were also marked as problematic due to their binding pose depending on a chain from the kinase being in a protein-protein complex rather than a holo structure as in the 
other members of the pocket. The final filtered datasets were composed of 4,260 and 820,280 protein-ligand pairs for the redocking (PDBbind ${ }^{[18}$ ) and cross-docking (Wierbowski et al. ${ }^{50}$ ) datasets, respectively.

Due to the combinatorial size of the cross-docking dataset, more filtering was required to make computational time tractable. Each unique protein binding pocket can be used to group the protein-ligand complexes as all of the receptor structures in the group share a common pocket. These groupings allow the cross-docking dataset to be downsampled for each pocket. Each pocket was either kept in full, or reduced to a random sample of 100 receptor-ligand pairs, whichever was smaller. We then removed any of the ligands that were previously listed as problematic. The downsampling results in 7,970 protein-ligand pairs and 92 unique protein binding pockets in the cross-docking dataset, where in no case is a ligand paired to its cognate receptor. Notably, 2 pockets were removed (ALDR and CP3A4) due to all of the ligands either failing the filtering, or being marked as problematic. We evaluate the docking performance differences for various fractions of the total complexes per pocket for both Gnina and Smina to ensure the downsampling does not bias the performance of either software (Figure S1.

\section{Evaluation Metrics}

TopN: We evaluate docking performance by examining the output poses. The Open Babel obrms tool $\frac{42[43}{4}$ is used to determine the RMSD from each output pose to the binding pose. In redocking, the binding pose is defined by the crystal pose. In cross-docking, we define the binding pose as the conformation of the ligand when the cognate protein structure is aligned with the reference structure. If the RMSD to the known binding pose is less than $2 \AA$ then we consider the pose to be "good." The percentage of systems with a good pose ranked in the top $\mathrm{N}$ (TopN) is reported for redocking. In the case of cross-docking, we consider all poses of a ligand across an ensemble of non-cognate receptors. We calculate the TopN for each target individually and report the average across the 92 systems. Averaging is performed to avoid 
over-representing targets with a large number of ligands. This metric can be computed for any number of output poses, computing only the top pose is Top1.

Avg Time Per System: Properly benchmarking the time for the various CNN scoring options on both the redocking and cross-docking datasets would take a significant amount of time, so we utilize a filtered version of the PDBbind v.2016 $6^{54}$ core set to determine runtime. This set consists of 263 complexes. The average runtime is calculated per system using the hyperfine benchmarking tool, $\sqrt[55]{5}$ computing the runtime for a minimum of 5 docking runs for each system (additional runs are automatically performed by hyperfine if high variability is encountered). We then average docking runtime over the whole PDBbind core set, to provide the average time for one docking run of the PDBbind core set. Timing evaluations were done on a dual 16-core $2.3 \mathrm{GHz}$ Intel Xeon 5218 with $96 \mathrm{~GB}$ of RAM and a $11 \mathrm{~GB}$ RTX $2080 \mathrm{Ti}$ GPU using CUDA 10.2 and cuDNN 7.6.5. All benchmarking runs were done on an otherwise unloaded system with four cores requested $(\mathrm{cpu}=4)$.

\section{Default Model Selection}

When using CNN scoring, the user can utilize a single CNN model or an ensemble of CNN models where the final score is an average of each CNN model's score. It has been shown that ensembles of predictors improve performance over a single predictive model.$[56$ However, due to the high computational cost of applying CNN models in comparison to empirical scoring functions, it is desirable to select a subset of the available models that provides improved scoring while limiting computational cost. The default CNN model ensemble was selected using a greedy forward algorithm. The ensemble was built in an iterative process using the "rescoring" option for CNN scoring so as to minimize the computational time for each iteration. In each round of the selection, models were chosen for their Top1

performance. We evaluate all of the versions of each CNN model (i.e. dense, dense_1, dense_2, dense_3, dense_4). In the first round of selection, all of the CNN models were tested for their individual ability to predict Top1 on both the cross-docking and redocking datasets. 
The next round of selection required testing of all two-model combinations with the model selected in the first step. Model selection continued, exploring all possible combinations of the built-in CNN models. The selection process concluded after five CNN models were selected for inclusion in the default ensemble.

\section{Default CNN Scoring Method}

GNINA allows the usage of CNN scoring in various steps of the molecular docking process (Figure 1). The CNN scoring option allows the user to change how the CNN is used to evaluate a ligand pose. If the CNN is not used at all in the scoring process ("none" option), then the molecular docking pipeline is essentially the same as SminA. The "rescoring" option has the lowest computational cost of the options that utilize the CNN models. With this option CNN models are used to score and re-sort the ligand conformations selected and refined by the non-CNN scoring function, defaulting to the Vina scoring function. For additional computational cost, the "refinement" option can be specified to use the CNN models for the refinement of the ligands after they have been selected by the Monte Carlo chains. In addition to refining the ligand conformations, the CNN models are used to score and resort the output poses, as the "rescore" option does. However, the Monte Carlo chains continue utilizing the non-CNN scoring function. The "all" option uses the CNN models as the scoring function throughout the course of the molecular docking procedure and has the highest computational cost by orders of magnitude. The CNN model is used for the selection process within the Monte Carlo chains, the refinement process after Monte Carlo selection, and the scoring and resorting of the poses before output. This option is very computationally intensive as the CNN is regularly queried for the energy of a particular conformation during the Monte Carlo sampling procedure.

In addition to the values allowed for cnn_scoring, the user is provided with cnn_empirical_ weight to combine the non-CNN and the CNN scoring functions. Using mix_emp_force the refinement of the ligand poses can be computed with a linear combination of the CNN gradi- 
ents and the non-CNN scoring functions force. mix_emp_energy uses the same linear combination of the scoring functions for computing the score of a given pose. The weighting of the Vina scoring function within the linear combination is selected with cnn_empirical_weight (default 1.0).

The default usage of the CNNs within the GNINA docking pipeline demands high accuracy while limiting computational costs. Therefore, each of the CNN scoring options is evaluated for both docking performance and runtime. Docking performance can be calculated on both the redocking and cross-docking datasets via TopN. To this end, GnINA is used with one of the CNN scoring options with the default CNN ensemble selected above to compute 9 ligand poses for each protein ligand system. We also investigate various values of cnn_empirical_weight while using the "refinement" option to determine if a linear combination of the non-CNN and CNN scoring functions provides greater docking performance. We use both mix_emp_energy and mix_emp_force with the cnn_empirical_weight parameter to use the linear combination of Vina and the default CNN ensemble for both refinement of poses and final scoring.

\section{Parameter Exploration}

Gnina has many parameters that alter the molecular docking pipeline. A default value is found for each of these parameters to provide the best all around default behavior. In exploring the various values of the parameters we find the optimal value for both the redocking and the cross-docking datasets. Additionally, we also consider that the user may not know the exact location of the binding pocket on the receptor and may have to use the entire protein as the autobox for the docking pipeline. Therefore, our setting exploration considers the case in which the specific binding pocket is known and the case in which the whole protein is used to define a binding box. Some parameters directly impact the sampling procedure, such as exhaustiveness, autobox_add, num_modes, num_mc_saved, and min_rmsd_filter which are described below. These parameters control, to some extent, the extensiveness of the search 
during the Monte Carlo sampling procedure. As previously stated, exhaustiveness determines the number of Monte Carlo chains run during the sampling procedure. autobox_add increases the size of the binding box that the Monte Carlo chains sample. num_modes determines the number of ligand poses output by GNINA at the completion of the docking procedure. This is separate from num_mc_saved which defines the number of ligand poses saved for each Monte Carlo chain. The number of ligand poses retained after all of the Monte Carlo chains are completed is determined by either the number of modes or the number of Monte Carlo saved, whichever is larger. After all of the Monte Carlo chains have completed and the poses have been refined and sorted, the RMSD between all pairs of ligand poses is calculated. min_rmsd_filter removes one pose from a pair if the RMSD of the pair is less than the value of the parameter. This ensures the poses returned by the docking procedure are all different from one another. When using the CNN scoring function, another setting is how many different rotations of the protein-ligand complex the CNN is able to see for each conformation, cnn_rotation.

Evaluations for all of the parameters are carried out on both the redocking and crossdocking datasets. Each setting is varied individually using the default CNN ensemble determined above. The values explored for each parameter are defined in Table 1 and Table 2. Values were explored around the previously set defaults for each of the parameters. Each value produced a set of poses, which was used to calculate a TopN.

Table 1: Parameters explored when the binding pocket has been defined

\begin{tabular}{|c|l|c|}
\hline Argument & Description & Values Explored \\
\hline exhaustiveness & Number of Monte Carlo chains & $4,8,16$ \\
\hline autobox_add & Increase size of binding box & $2,4,6,8$ \\
\hline num_modes & Number of output conformations & 9,100 \\
\hline num_mc_saved & $\begin{array}{l}\text { Number of conformations saved from each } \\
\text { Monte Carlo chain }\end{array}$ & $20,40,60,80,100$ \\
\hline min_rmsd_filter & Minimum RMSD to filter saved poses & $0.5,1.0,1.5$ \\
\hline cnn_rotation & Number of rotations of data to show the CNN & $0,1,5,10,20$ \\
\hline
\end{tabular}


Table 2: Parameters explored when the binding pocket is not known and the whole protein is used for docking

\begin{tabular}{|c|c|c|}
\hline Argument & Description & Values Explored \\
\hline exhaustiveness & Number of Monte Carlo chains & $8,16,32,64$ \\
\hline
\end{tabular}

\section{CNN Scoring Performance}

All of the CNN models were trained on some subset of the cross-docking and redocking datasets. Generalization can be evaluated by determining the performance of the CNN scoring functions on the subset of the datasets that were not seen in training. This evaluation is carried out by removing any protein or ligand contained within the training data of the CNN models. All of the CNN models have been trained on different sets, so fully testing generality requires the removal of all of the proteins and ligands in the training set from both the redocking and cross-docking datasets. We removed all PDB IDs that were in the training sets of the CNN models, ${ }^{[1 / 45}$ leaving 441 and 178 protein-ligand pairs for the redocking and cross-docking sets, respectively.

The CNN models output both a CNNscore and a CNNaffinity for each of the conformations output by GNINA. CNNaffinity is the affinity of the docked complex as determined by the CNN, this metric has been evaluated in a previous work. ${ }^{45}$ The CNNscore is a value between 0 and 1 that is used to rank the poses of the ligand, where a score of 1 denotes a perfect ligand pose. We would like to investigate if there is a correlation between high scores and low RMSD to the crystal pose. For ease of analysis, we only consider the top ranked pose. Using the top ranked pose for each complex, we investigate how filtering the poses by their CNNscore can affect the percentage of poses in which the RMSD to the crystal pose is less than $2 \AA$. 


\section{Results}

\section{Smina Comparison}

GNINA is a fork of SMINA that allows the utilization of CNN models as scoring functions. Therefore, without the use of the CNN models GNinA should function exactly as SMINA does. However, unlike Smina, GninA does computation with single (32 bit) precision rather than double (64 bit) precision due to the need to shift calculations to the GPU for efficient CNN scoring. Therefore, we ensure that the use of single precision does not negatively affect the docking power of the pipeline. The effect of this precision change can be evaluated by running GNINA without using any CNN scoring and with autobox_extend turned off, allowing us to compare to SMINA docking results. We consider only redocking results as cross-docking results would require significantly more computation, and identification of differences due to precision can be done using only redocking results. Results for redocking do not show a significant difference for the output poses. A majority of the output poses are exactly the same, with slight differences seen for some output poses (Figure S2 and S3).

\section{Default Model Selection}

The iterative process for construction of the default CNN ensemble, denoted Default Ensemble, is shown in Table[S1. The five selected models are dense, general_default2018_3, dense_3, crossdock_default2018, and redock_default2018. We now evaluate the docking performance boost that this ensemble provides over any single CNN model type (e.g. crossdock_default2018, dense, etc.) or an ensemble of the same CNN model type (e.g. crossdock_default2018_ensemble, dense_ensemble, etc.). We compare the CNN model(s) docking performance by evaluating TopN on both the redocking and the cross-docking tasks. The CNN models are used in the "rescoring" option for the CNN scoring to output 9 ligand conformations.

The docking performance of the Default Ensemble is compared to the single model options 


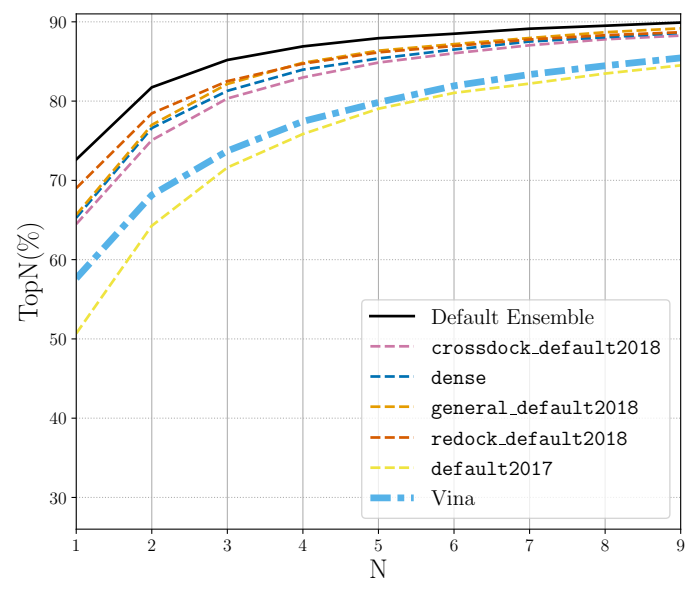

(a) Redocking

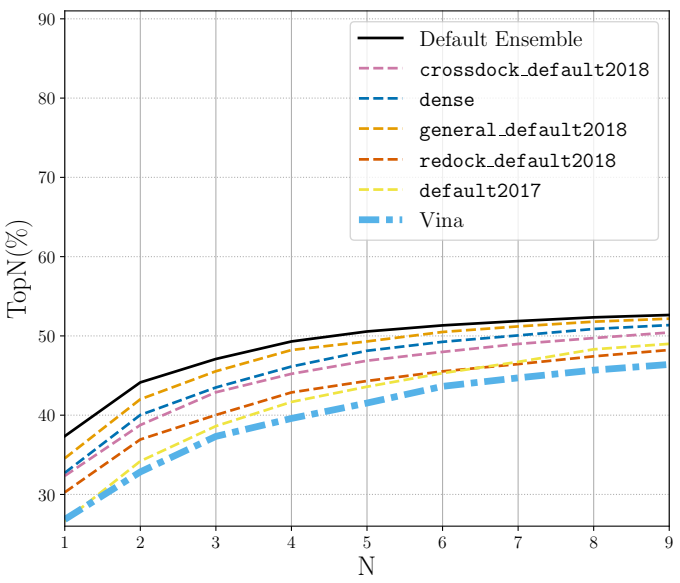

(b) Cross-docking

Figure 2: Docking using the single CNN models and the newly selected Default Ensemble for rescoring the output poses. The binding pocket is defined by the known binding ligand. TopN is the percentage of targets ranked above or at $N$ with a RMSD less than $2 \AA$.

in Figure 2. While nearly all models are able to outperform Vina, we can see that the newly selected Default Ensemble significantly outperforms all of the single models on both the redocking task and the cross-docking task.

When the Default Ensemble is compared to the ensembles of each of the individual CNN model types, we see that the Default Ensemble is able to outperform all of the ensembles composed of one model type (Figure 3). The ensemble selection procedure determined five CNN models whose combined performance on ranking low RMSD poses first beats the performance of the ensemble utilizing all of the built-in models while significantly reducing computational cost. The All Ensemble is the ensemble composed of all 16 CNN models builtin to the Gnina software. The Default Ensemble is able to meet the docking performance of this large ensemble, even when considering cross-docking results per pocket (Figure S4), while only being composed of five models. Reducing the number of models in the ensemble enables the computations to be several seconds faster for an average docking run (Figure 4). This reduction is likely due to the inclusion of only two of the dense models which take the longest to run because of the high number of parameters in the models. The computational 
speedup afforded by the Default Ensemble over the All Ensemble increases when no GPU is used for docking (Table S2). The computational speed boost can have a significant impact when performing a large number of docking runs or when there is no GPU available for enhanced parallelism of the scoring computation.

When comparing Figure 2 and Figure 3 we can see that the ensembles composed of the individual model types are able to outperform their single model counterparts. We therefore omit the single models for the remaining evaluations.

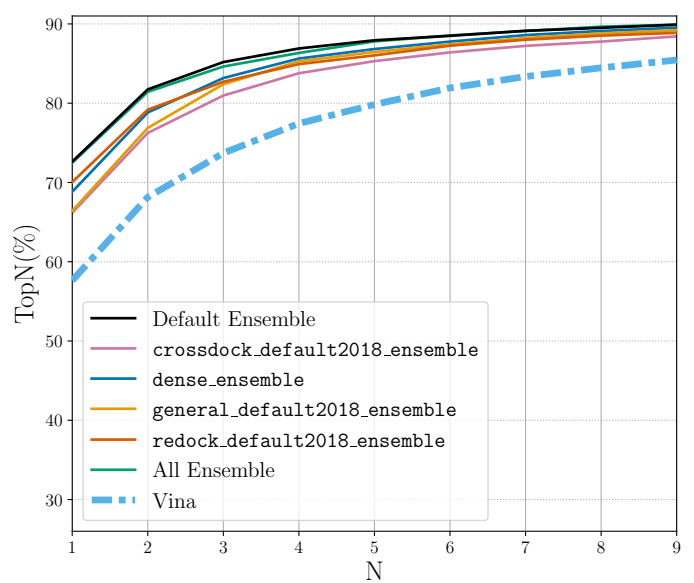

(a) Redocking

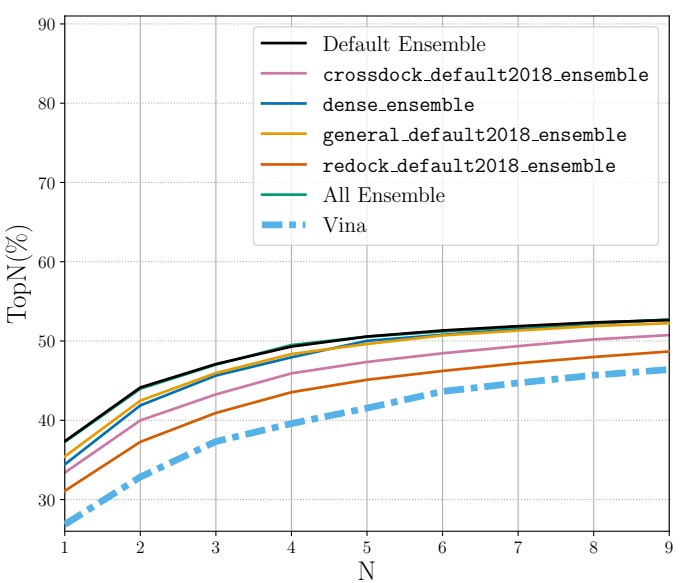

(b) Cross-docking

Figure 3: Docking using the ensemble of each type of CNN model, the full ensemble of CNN models, and the newly selected Default Ensemble for rescoring the output poses. The binding pocket is defined by the known binding ligand. TopN is the percentage of targets ranked above or at $\mathrm{N}$ with a RMSD less than $2 \AA$.

\section{Default CNN Scoring Method}

We evaluate the performance of the Default Ensemble with the "rescore," and "refinement" options of cnn_scoring. The usage of the "all" option was unable to complete on the PDBbind Core set in a reasonable amount of time, so it was not considered. The "all" option requires usage of the CNN scoring function for every mutation of the ligand within every Monte Carlo chain with each usage of the CNN scoring function having a high computational 


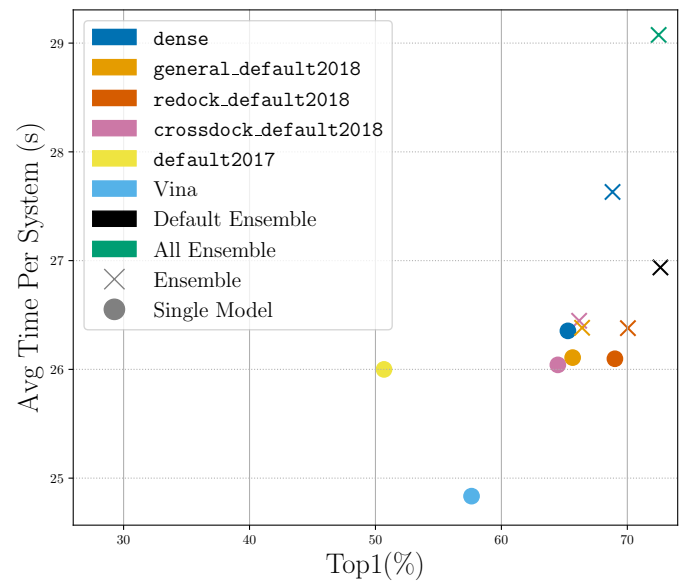

(a) Redocking

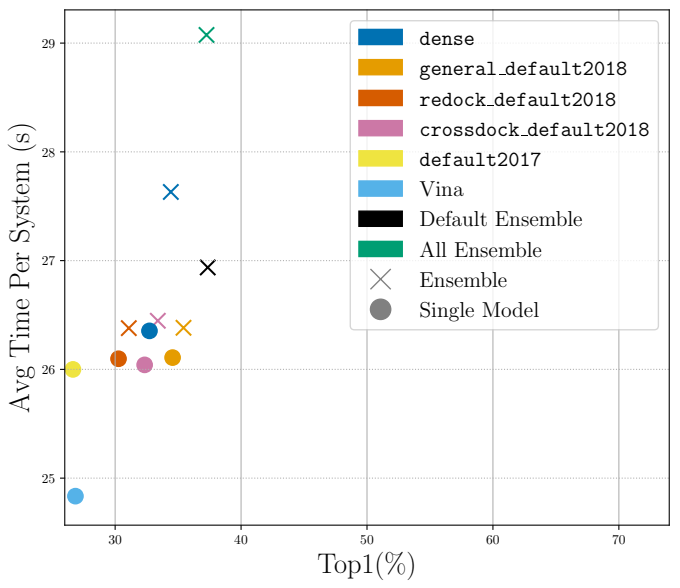

(b) Cross-docking

Figure 4: Evaluation of the average time to dock one protein-ligand system from the PDBbind core set v.2016. Top1 is the percentage of top ranked targets with a RMSD less than $2 \AA$.

cost. This leaves us with the "refinement" and "rescoring" options of cnn_scoring. Figure 5 shows the Default Ensemble performs nearly as well with either option. We can also see that using cnn_emp_weight with both mix_emp_energy and mix_emp_force does not significantly alter the docking performance when using the "refinement" option (Figure S5).

However, from looking at the average time to perform molecular docking for one system we see that "refinement" takes an order of magnitude longer than "rescoring" (Figure S6). Time for performing "rescoring" on an average system is similar to the time to perform docking with the Vina scoring function. We find it reasonable to use "rescore" as the default option for the CNN scoring due to its docking performance and runtime.

\section{Parameter Exploration}

Changes in the exhaustiveness alter the amount of sampling that occurs. When the exhaustiveness is increased, we see an increase in the performance of docking (Figure 6). This is as expected as more Monte Carlo chains randomly mutating the ligand conformation provides the docking procedure with more opportunities to randomly sample the correct pose. 


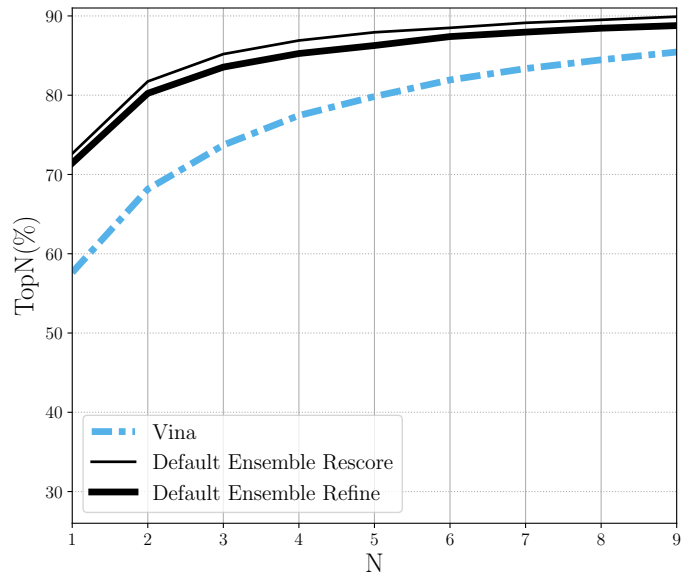

(a) Redocking

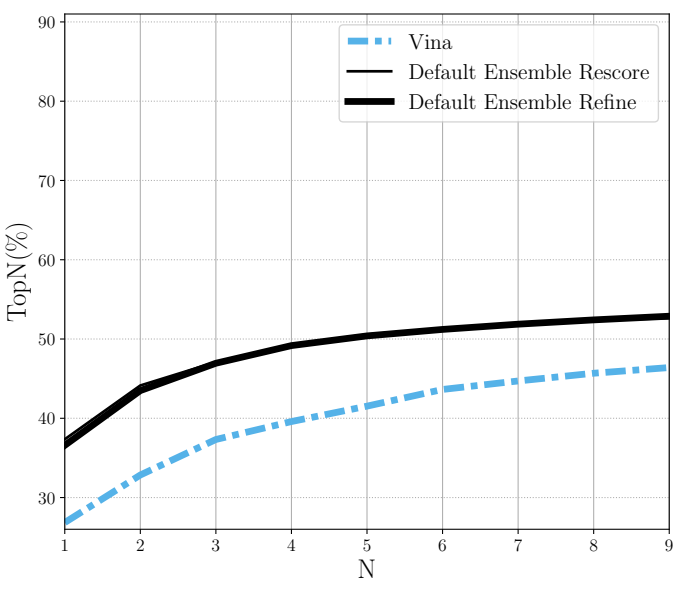

(b) Cross-docking

Figure 5: Comparing the Default CNN Ensemble for use in only rescoring of the poses output by the Monte Carlo chains or the refinement of the poses followed by a rescoring of the poses. The "refine" option has nearly the same docking performance as the "rescore" option when cross-docking. TopN is the percentage of targets ranked above or at $\mathrm{N}$ with a RMSD less than $2 \AA$.

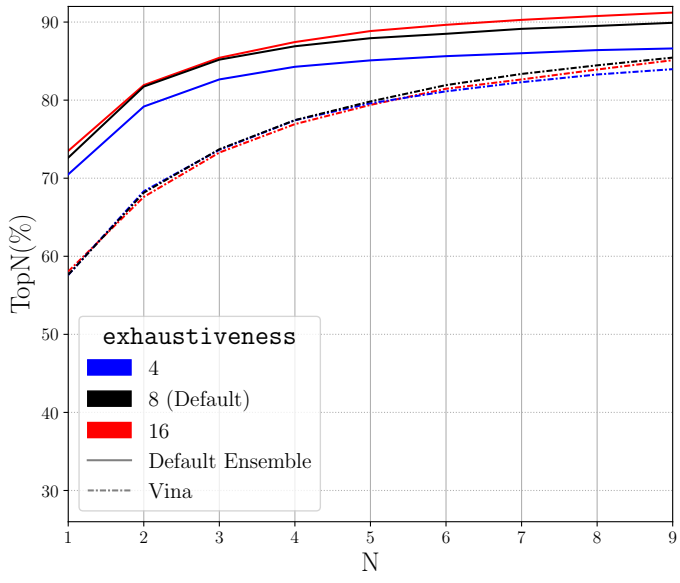

(a) Redocking

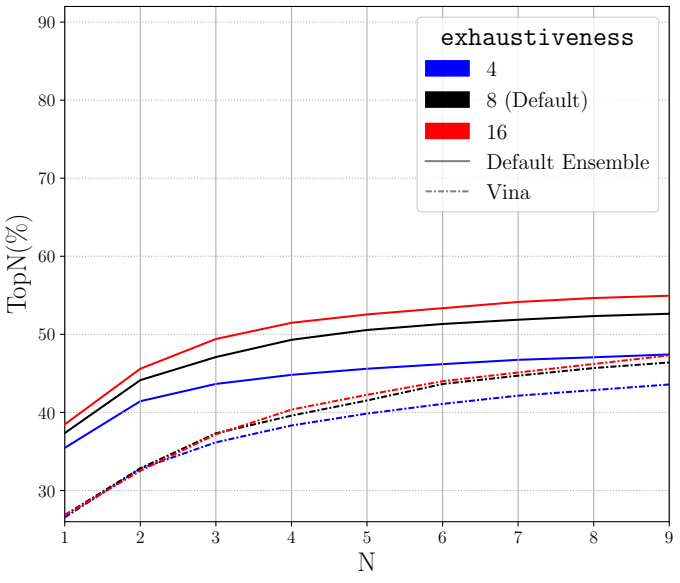

(b) Cross-docking

Figure 6: Evaluating the role of exhaustiveness in the performance of docking with the Default CNN Ensemble by analyzing TopN. TopN is the percentage of targets ranked above or at $\mathrm{N}$ with a RMSD less than $2 \AA$. 
However, there are no significant performance gains after a value of 8 . An exhaustiveness of 16 provides some performance boost, but this boost may be accompanied by a doubling of the computational time. The Monte Carlo chains are evaluated in parallel, but parallelism is limited by the number of cores available to GNINA. If the exhaustiveness is greater than the CPUs provided to GninA, the number of simultaneously running Monte Carlo chains is equal to the number of CPUs. Therefore, in typical usage of GNinA, an exhaustiveness level of 8 is sufficient for adequate performance levels while minimizing the computational load when targeting a specific binding site.

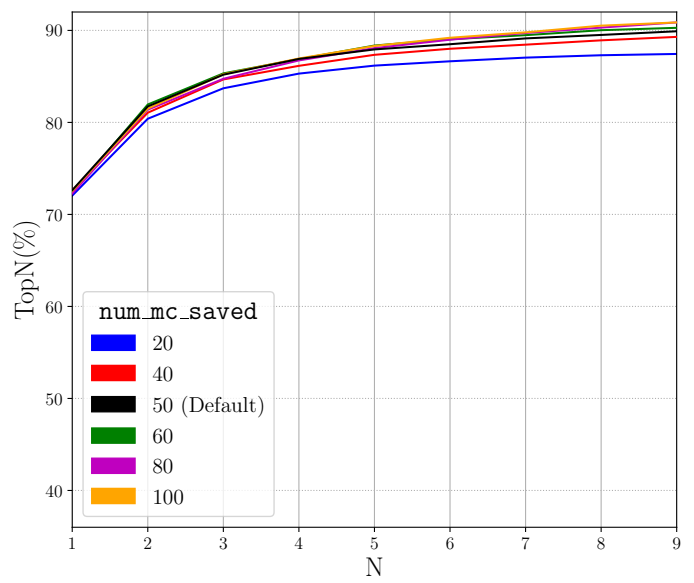

(a) Redocking

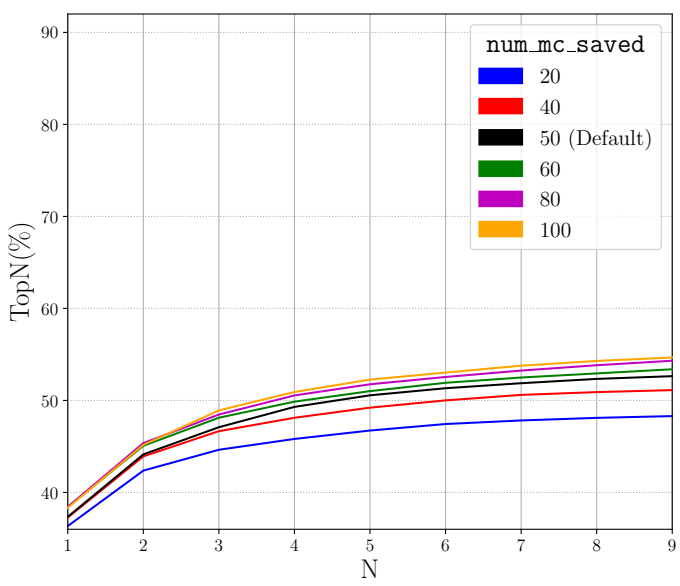

(b) Cross-docking

Figure 7: Evaluation of the Number of Monte Carlo Saved in the performance of docking with the Default CNN Ensemble by analyzing TopN. TopN is the percentage of targets ranked above or at $\mathrm{N}$ with a RMSD less than $2 \AA$.

Increasing the num_mc_saved parameter increases the chances of sampling accurate docking poses, as increasing the number of output conformations from each Monte Carlo chain increases the likelihood of finding the correct pose. However, this will also increase computational time due to the fact that more poses require refinement and final scoring. As num_mc_saved gets closer to 100, we see that the docking performance boost is reduced. Therefore, we select a new default value of 50 for num_mc_saved to minimize the computational overhead while still increasing the performance of the docking routine. The 
num_mc_saved and num_modes affect one another; the number of poses saved from each Monte Carlo chain is the maximum of the two values. When looking at the first 9 poses, we see an increase in the docking performance with a substantially greater value for the number of output poses (num_modes). This is due to num_modes forcing each Monte Carlo chain to output a number of poses greater than num_mc_saved. Increasing the default value of num_modes to a value higher than 50 (the default value for num_mc_saved) will again increase computational overhead, so the default value is set to 9 .

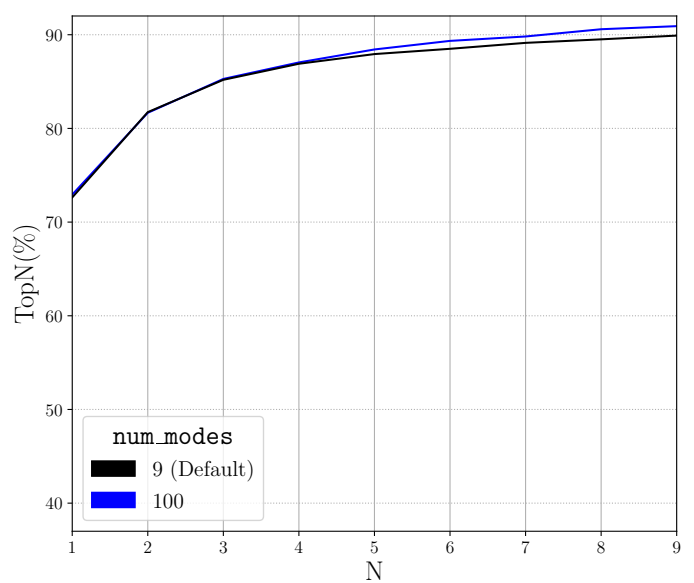

(a) Redocking

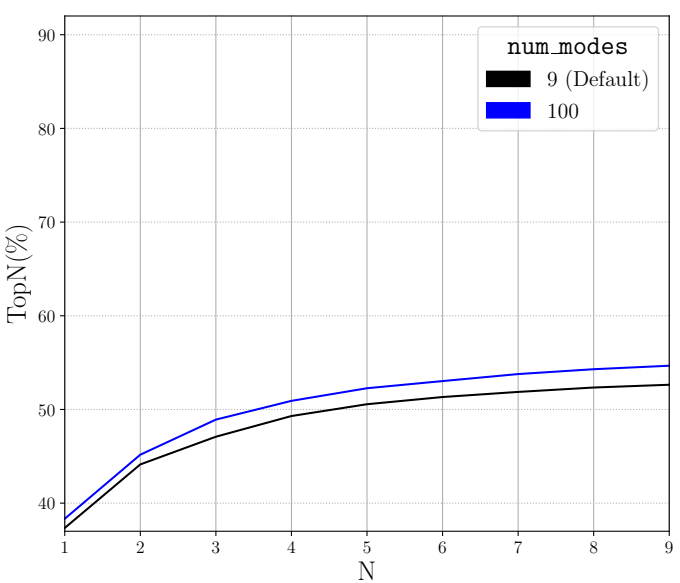

(b) Cross-docking

Figure 8: Evaluating a much greater number of modes on the performance of docking with the Default CNN Ensemble by analyzing TopN. TopN is the percentage of targets ranked above or at $\mathrm{N}$ with a RMSD less than $2 \AA$.

We next evaluate the size of the targeted binding site (box). The autobox_add parameter increases the search space for the docking program to be larger than the rectangular prism defined by the autobox_ligand input to GNINA. In redocking (Figure S7a), the expansion of the search space decreases performance as the expanded search space increases the potential conformational space of the ligand. A higher value of autobox_add is necessary to ensure that the correct binding site, which may differ from the binding site of the autobox_ligand, is included in the search space. This is shown during cross-docking (Figure S7b), where only the binding site of the cognate ligand is known. A low value of autobox_add artificially 
improves docking performance when the binding pose is known by unrealistically constraining the search space. We therefore select a default value of 4 for autobox_add to keep the box small while still providing room for error in the selection of the binding site.

Changes to the CNN rotations do not significantly change the scoring of the Default Ensemble (Figure S8). The CNN Ensemble is able to determine the correct score for the ligand pose regardless of the rotation of the ligand and protein complex. Altering the value of the minimum RMSD filter does not change the results of the docking (Figure S9). Filtering out poses with similar conformations increases the diversity of poses that the CNN ensemble ranks. However, the CNN ensemble is able to accurately rank the poses it sees, providing high scores to poses with low RMSD to the known binding pose.

\section{Whole Protein Docking}

Next, we evaluate the performance of docking when using the whole protein as the defined binding site. Whole protein docking can be used for new protein targets when the true binding site is unknown. The performance of docking is expected to be reduced as the sampling space has been significantly increased. When using the whole protein for the sampling space, the ligand tends to get stuck at local energy minima which are distant from the actual binding site. Most of the protein surface is not hospitable for ligand binding, so once a potential pocket is discovered, exploring more of the protein surface has a low probability during the Monte Carlo sampling procedure. Comparing Figure 9 to Figure 6 shows that the docking performances of both Vina and the Default CNN Ensemble are reduced from when the binding pocket was explicitly defined. Redocking performance decreases from $73 \%$ to $38 \%$ at Top1 with exhaustiveness at 8 while cross-docking decreases from $37 \%$ to $16 \%$ at Top1 with exhaustiveness at 8 (cross-docking per pocket Top1 is shown in Figure S10). The larger potential docking space requires more sampling to find a ligand conformation with low RMSD to the known binding pose. As expected, with whole protein docking we see greater increases in performance with increased sampling (exhaustiveness). By again 
comparing Figure 9 with Figure 6 we see that this boost in performance is larger than when the binding pocket is defined explicitly and does not exhibit the same diminishing returns. When increasing exhaustiveness from 8 to 16 in whole protein docking Top9 increases from $48 \%$ to $58 \%$ and $22 \%$ to $29 \%$ for redocking and cross-docking, respectively. If the binding pocket is explicitly defined, the same change in exhaustiveness only increases Top9 from $87 \%$ to $88 \%$ and $53 \%$ to $55 \%$ for redocking and cross-docking, respectively. For this reason, when performing whole protein docking, we recommend setting the value of exhaustiveness as high as possible given time constraints.

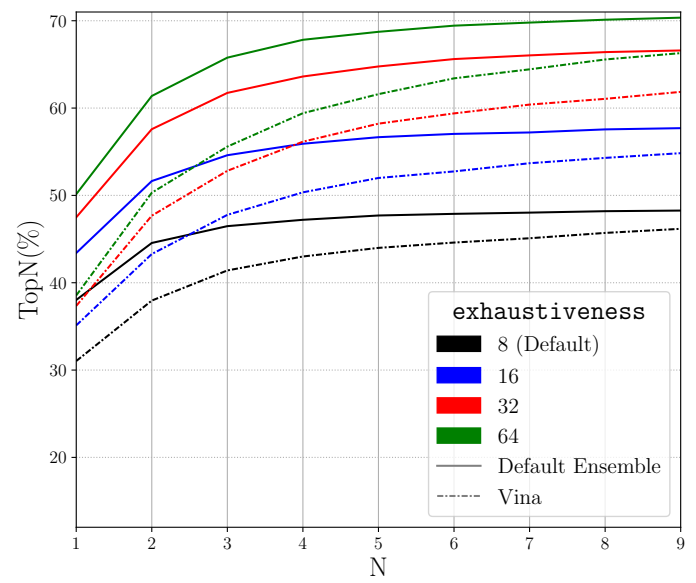

(a) Redocking

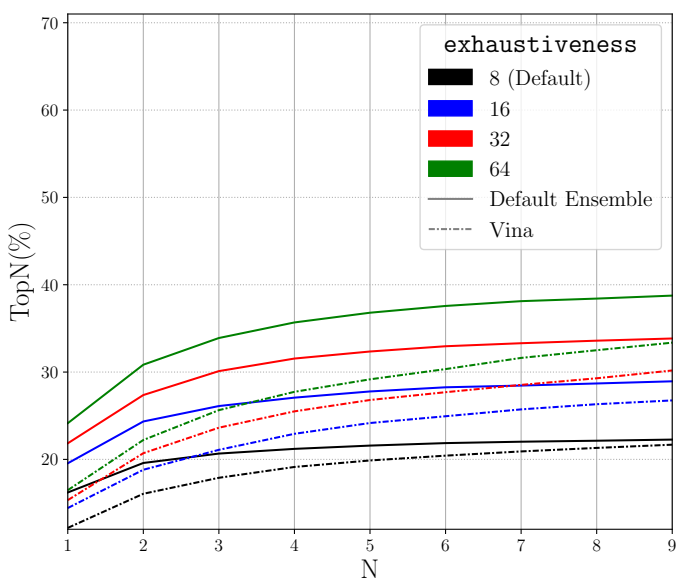

(b) Cross-docking

Figure 9: Increasing the exhaustiveness when using the whole protein as the binding box. TopN is the percentage of targets ranked above or at $\mathrm{N}$ with a RMSD less than $2 \AA$.

\section{Flexible Docking}

We now test the performance of GNinA for docking with flexible side chains. Since receptor flexibility is only useful in the context of cross-docking, we limit our tests to the crossdocking dataset; allowing side-chain re-arrangements would only deteriorate the performance in redocking given that the receptor is already in the correct conformation.

Flexible docking is computationally more expensive than docking with a rigid receptor 
because of the larger number of degrees of freedom to be sampled. For this reason, we only use the default parameters carefully selected above. In order to define the side chains to be treated as flexible, autobox_ligand is also used as flexdist_ligand and flexdist is set to $3.5 \AA$, which gives a reasonable representation of the protein-ligand binding site. ${ }^{57}$ Therefore, conformations for all side chains with at least one atom within $3.5 \AA$ from flexdist_ligand are sampled during docking.

Out of the 7970 systems in the cross-docking dataset, flexible side chains are not identified for 24 protein-ligand complexes (see Table S3 for details). Such systems are discarded from the following analysis of flexible docking since they are equivalent to rigid docking. Additionally, 9 systems were problematic when computing RMSDs for the flexible residues because of connectivity issues. Disulfide bonds between cysteine residues are allowed to break during sampling (with a software warning), which results in a different connectivity in the output file for four systems. In the other five systems, the connectivity between flexible residues in the input and output file was found to be different and therefore they were discarded (see Table $\mathrm{S} 3$ for details). All nine systems with connectivity issues were removed from the analysis, resulting in a dataset of 7937 cross-docked complexes.

In order to assess the performance of flexible docking compared to rigid docking, we look at RMSD differences between ligands docked with both methods in relation to the similarity between binding pockets of the cognate and docking target receptors. The similarity of the binding pockets is assessed via side chains RMSD between the docking target (non-cognate receptor) and the cognate receptor, which we denote target-cognate RMSD. This is distinct form either the side chain RMSD between docking input and output (target-pose RMSD) and the side chain RMSD between cognate receptor and docking output (cognate-pose RMSD). The target-cognate RMSD is computed by finding the best match between residues in the target and cognate receptors that are within $3.5 \AA$ from the ligand being docked.

Figure 10a shows the difference in RMSDs for the ligand top pose between flexible and rigid docking versus the target-cognate RMSD. For higher target-cognate RMSDs, indicating 
differences in the binding pocket between the target and cognate receptor, one would expect flexible docking to perform better. However, as we can see, the difference in ligand RMSD between flexible and rigid docking for the top pose varies widely between systems and there in no clear advantage in flexible docking. The overall RMSD distributions for the ligand top poses (Figure S11) are fairly similar, with slightly more systems with low RMSD for rigid docking than flexible docking.

Figure $10 \mathrm{~b}$ shows the average RMSD difference between rigid and flexible docking for different $1 \AA$ intervals of target-cognate side chains RMSDs. For low target-cognate RMSDs, which correspond to a highly similar binding site and therefore a situation akin to redocking, rigid docking seems to be advantageous on average, as expected. In the other hand, for higher target-cognate side chains RMSDs, indicating a lower similarity between binding sites, the situation is less clear. Flexible docking seems to be equivalent or slightly more advantageous, on average, especially at higher target-cognate side chains RMSDs. However, as it can be inferred from Figure 10a, the number of systems with target-cognate side chains RMSD higher than $6 \AA$ is low and therefore the apparent improvement in ligand RMSD for flexible docking is inconclusive.

When performing flexible docking, the side chains identified as flexible are included in the calculation of the bounds for the box defining the search space. This results in a larger search space for flexible docking compared to rigid docking, which in turn might require a higher exhaustiveness for better sampling (although by default the number of Monte Carlo steps is already proportional to the number of degrees of freedom, hence the increased computational cost of flexible docking). It is also worth stressing that we used the default CNN models, which were not explicitly trained for flexible docking.

Given the much higher computational cost of flexible docking, the optimization of GNINA default parameters and training of new CNN models for this specific task is outside the scope of the present work and will be addressed in future versions of GnINA. However, it is clear that improvements for flexible docking are system-dependent and, therefore, accounting for 


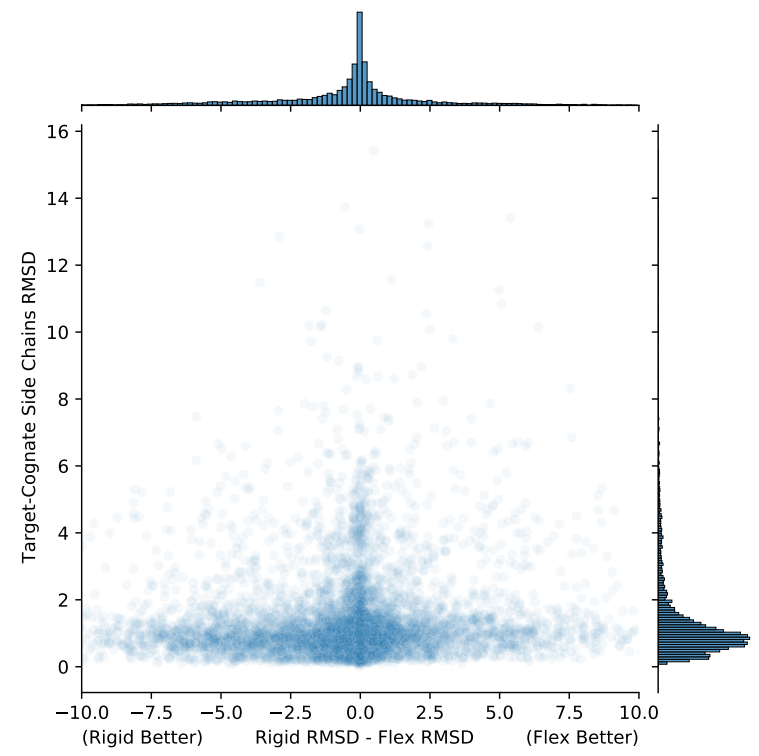

(a)

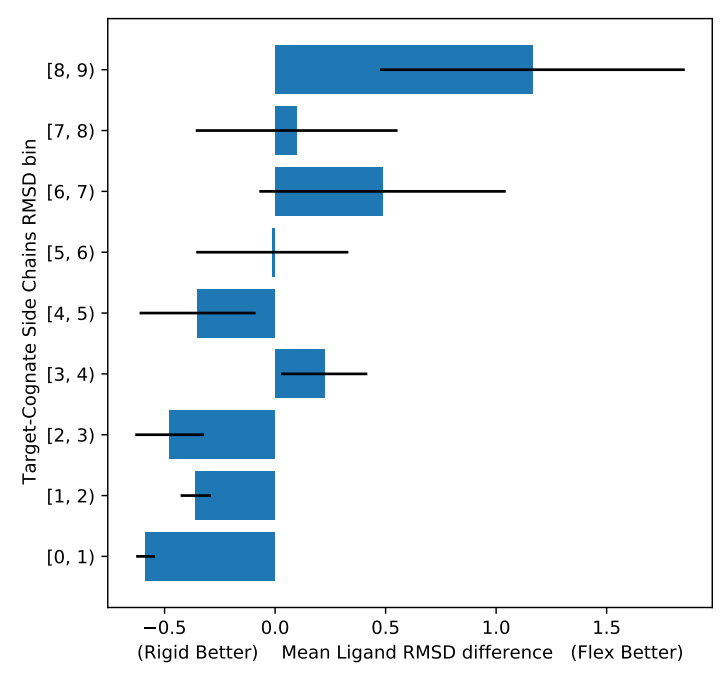

(b)

Figure 10: Comparison between rigid and flexible docking with the default GNINA parameters: (a) ligand RMSD differences between rigid and flexible docking versus target-cognate side chain RMSDs, (b) average ligand RMSD difference for different $1 \AA$ intervals of targetcognate side chains RMSD.

the increased computational cost, it is reasonable to use rigid docking as the default docking method.

\section{CNN Scoring Performance}

We evaluate all of the CNN models on a subset of the data that was not seen during training to ensure that the CNN models are able to generalize to unseen protein-ligand systems. We also show the Vina results for the same subset of protein-ligand systems. The CNN models' docking performance decrease relative to the full sets when looking at the top pose, (comparing Figure 11 to Figure 3). Redocking Top1 performance drops from $73 \%$ to $68 \%$ on the Default Ensemble for the filtered set and the full set, respectively, while Vina remains at about 57\%. Cross-docking Top1 performance increases from 37\% to $42 \%$ for the Default Ensemble and decreases from $27 \%$ to $23 \%$ when using the Vina scoring function. Overall, the Default Ensemble still shows a strong docking performance boost when used to rescore poses 
rather than using the Vina scoring function to score poses even on protein-ligand systems not seen during training.

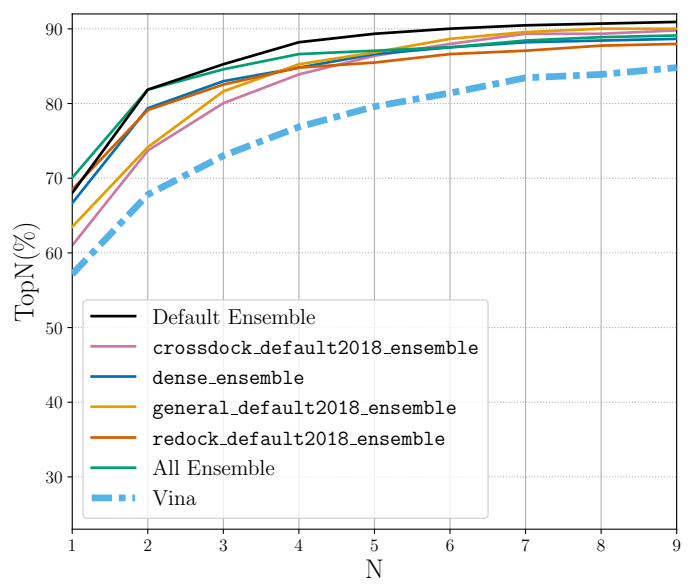

(a) Redocking Ensembles

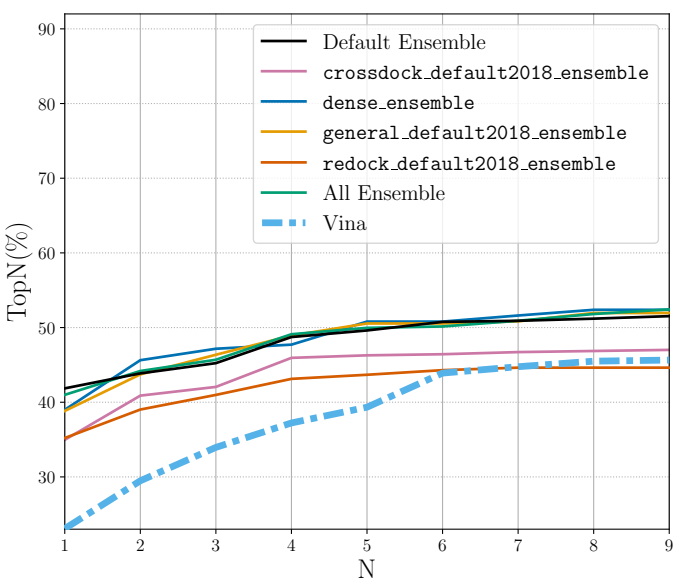

(b) Cross-docking Ensembles

Figure 11: CNN model ensembles evaluated on the subset of proteins and ligands not present in their training datasets. Ensemble models used with the default arguments defined above. TopN is the percentage of targets ranked above or at $\mathrm{N}$ with a RMSD less than $2 \AA$.

Finally, we examine the importance of the output CNNscore as a measure of the confidence of the prediction.

We show in Figure 12 that poses with high CNNscores are more likely to be low RMSD to the known binding pose. However, when the CNNscore threshold is close to 1, each CNN ensemble has few poses remaining. Comparing the percentage of complexes remaining when thresholding by CNNscore, we can see that the CNN models are much more confident in the poses when they are performing the redocking task: $87 \%$ of systems have a top pose with a score higher than 0.8. All of the CNN ensembles identify fewer poses with a high CNNscore for the cross-docking task: only $15 \%$ of cross-docked ligands have a pose with a score higher than 0.8 (Figure $\mathrm{S12}$ ). In general, higher CNNscores imply that a pose is more likely to have a low RMSD. 

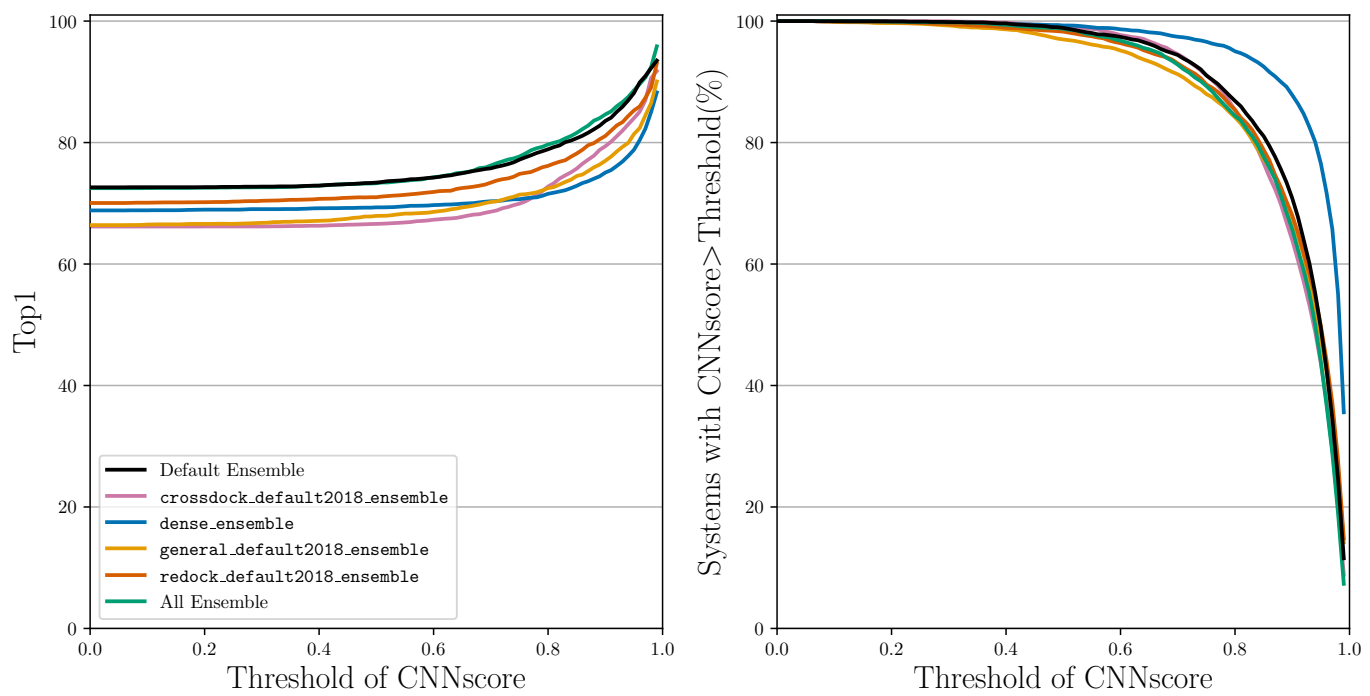

(a) Redocking Ensembles
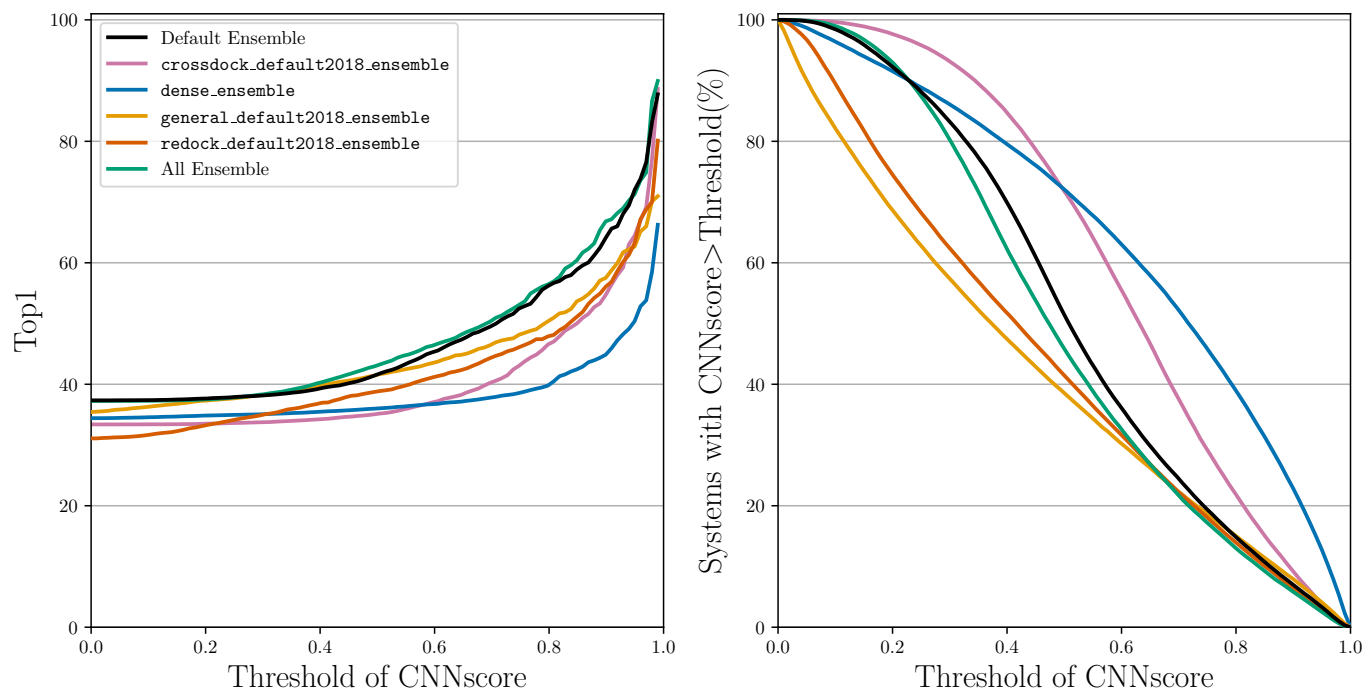

(b) Cross-docking Ensembles

Figure 12: Thresholding the top pose by the score determined by the CNN. Top1 is the percentage of top ranked targets with a RMSD less than $2 \AA$. 


\section{Discussion}

We show that our computational docking software GninA is able to outperform AutoDock Vina scoring by using CNN models to rescore generated poses. Without using a CNN model, our software is equivalent to using the SMINA docking software, which is a fork of Vina. Gnina allows the user to utilize CNN models as scoring functions within the docking pipeline in a variety of ways. The various CNN scoring options allow the specified CNN models to replace the scoring function in the sampling, refinement, and rescoring steps of the docking pipeline. We first establish an ensemble of built-in CNN models to be used as the default ensemble. This ensemble of models is selected for its docking and runtime performance. The selected ensemble, termed the Default Ensemble, is able to exceed the ranking performance of Vina (Top1 increases from $58 \%$ to $73 \%$ and from $27 \%$ to $37 \%$ for redocking and cross-docking, respectively) while only adding two seconds to the average compute time when utilizing GPUs. It also significantly outperforms any single model or any ensemble of identical models while being significantly faster than an ensemble of all models. The Default Ensemble performs almost equally well when performing refinement of the sampled poses rather than using the Vina scoring function. Refinement with the CNN models is not recommended as the compute cost is significant and the performance is less than simply using the CNN models to rescore the poses refined by the Vina scoring function.

We next derive the default parameters when using the Default Ensemble for docking with GninA. autobox_add increases the amount of space around the defined binding pocket to allow more volume for sampling to investigate. Increases in autobox_add decrease the accuracy of the predicted binding pose when the precise binding pocket is known. However, a higher autobox_add is necessary if the true binding site is not known. We find that increases in exhaustiveness, or the number of Monte Carlo chains run, boost the performance of the docking procedure at the cost of extra computation. We also show that our Default Ensemble with an exhaustiveness value of 8 (Top1 $73 \%$ on redocking and $37 \%$ on crossdocking) is able to outperform the Vina scoring function with double the exhaustiveness 
(Top1 $58 \%$ on redocking and $27 \%$ on cross-docking). This shows that using the Default Ensemble for rescoring is more valuable than doubling the amount of sampling of the docking procedure. Similarly, increasing the number of poses saved from each Monte Carlo chain, num_mc_saved, and the number of poses output by the docking pipeline, num_modes, increases the performance of the docking routine at the expense of increased computation. The value of the cnn_rotation and the value of min_rmsd_filter do not seem to alter the performance of the docking pipeline. The default arguments for GNINA when the binding pocket is explicitly defined are: autobox_add (4), exhaustiveness (8), num_mc_saved (50), num_modes (9), min_rmsd_filter (1.0), and cnn_rotation (0).

However, when the exact binding pocket is not known and the whole protein is used as the defined binding pocket we see that exhaustiveness has a much greater impact on performance. We see that the Default Ensemble with exhaustiveness 16 (Top1 43\% on redocking and $20 \%$ on cross-docking) is able to outperform the Vina scoring function even when the exhaustiveness is quadrupled to 64 (Top1 $38 \%$ on redocking and $16 \%$ on crossdocking). Again, this shows the importance of using the Default Ensemble for rescoring rather than increasing exhaustiveness with the Vina scoring function. The Default Ensemble still shows docking performance improvements with increasing exhaustiveness on both redocking and cross-docking when the exact binding pocket is unknown. We therefore recommend the largest value of exhaustiveness that can be used given time constraints when performing whole protein docking.

We tested the impact of performing cross-docking with flexible side chains (flexible docking). For high binding pocket similarity, flexible docking resulted in a slight deterioration of average ligand top pose RMSDs, as expected. However, for low binding pocket similarity there is no clear advantage for flexible docking, whose success is very much system-dependent. In light of this and given the much higher computational cost, rigid docking remains the most suitable default docking procedure.

Finally, we evaluate the ability of the CNN ensembles to score ligand conformations. In 
order to ensure that our CNN models are generalizing to unseen protein-ligand complexes, we filter the redocking and cross-docking benchmark datasets to only include protein-ligand pairs that were not seen during training of the CNNs. We show that our CNN model ensembles are able to outperform the Vina scoring function. The CNN models are able to generalize to unseen examples and properly score the ligand conformations such that the low RMSD poses are ranked higher more often than when using the Vina scoring function. The score output can provide a probability of a pose being less than $2 \AA$ from the binding pose. When the CNN outputs a score greater than 0.8 during cross-docking there is at least a $56 \%$ probability that the pose is less than $2 \AA$ RMSD from the correct pose. If redocking is performed, then this probability is $79 \%$. The score output by the CNN models can be used as an indicator of the confidence in the quality of the generated ligand conformation; however, particularly when cross-docking, high scoring poses are less common (Figure 12b).

The source code (https://github.com/gnina/gnina) and a pre-built docker container (https://hub.docker.com/r/gnina/gnina) for GNINA are available under a dual GPL2/ Apache License.

\section{Acknowledgement}

The authors thank Monica Dayao, Rosalie Nolen, and Jonathan King for their comments during the preparation of the manuscript. RM thanks Philip Biggin for fruitful discussions and support. We also thank Zelda, Lily, and Eddie for moral canine support. This work is supported by R01GM108340 from the National Institute of General Medical Sciences and is supported in part by the University of Pittsburgh Center for Research Computing through the resources provided.

RM is supported by funding from the Biotechnology and Biological Sciences Research Council (BBSRC) National Productivity Investment Fund (NPIF) [BB/S50760X/1] and Evotec (UK) via the Interdisciplinary Biosciences DTP at the University of Oxford [BB/MO11224/1] 
and was supported by the Google Summer of Code 2019 program.

\section{Supporting Information Available}

\section{References}

(1) Kitchen, D. B.; Decornez, H.; Furr, J. R.; Bajorath, J. Docking and scoring in virtual screening for drug discovery: methods and applications. Nature reviews Drug discovery 2004, 3, 935-949.

(2) Leach, A. R.; Shoichet, B. K.; Peishoff, C. E. Prediction of protein- ligand interactions. Docking and scoring: successes and gaps. Journal of medicinal chemistry 2006, 49, $5851-5855$.

(3) Lyu, J.; Wang, S.; Balius, T. E.; Singh, I.; Levit, A.; Moroz, Y. S.; O’Meara, M. J.; Che, T.; Algaa, E.; Tolmachova, K., et al. Ultra-large library docking for discovering new chemotypes. Nature 2019, 566, 224-229.

(4) Muegge, I.; Martin, Y. C. A general and fast scoring function for protein- ligand interactions: a simplified potential approach. Journal of medicinal chemistry 1999, 42, 791-804.

(5) Muegge, I. A knowledge-based scoring function for protein-ligand interactions: Probing the reference state. Perspectives in Drug Discovery and Design 2000, 20, 99-114.

(6) Brooijmans, N.; Kuntz, I. D. Molecular recognition and docking algorithms. Annual review of biophysics and biomolecular structure 2003, 32, 335-373.

(7) Huang, N.; Kalyanaraman, C.; Bernacki, K.; Jacobson, M. P. Molecular mechanics methods for predicting protein-ligand binding. Physical Chemistry Chemical Physics 2006, 8, 5166-5177. 
(8) Liu, J.; Wang, R. Classification of current scoring functions. Journal of chemical information and modeling 2015, 55, 475-482.

(9) Wang, R.; Lai, L.; Wang, S. Further development and validation of empirical scoring functions for structure-based binding affinity prediction. Journal of computer-aided molecular design 2002, 16, 11-26.

(10) Trott, O.; Olson, A. J. AutoDock Vina: improving the speed and accuracy of docking with a new scoring function, efficient optimization, and multithreading. Journal of computational chemistry 2010, 31, 455-461.

(11) Eldridge, M. D.; Murray, C. W.; Auton, T. R.; Paolini, G. V.; Mee, R. P. Empirical scoring functions: I. The development of a fast empirical scoring function to estimate the binding affinity of ligands in receptor complexes. Journal of computer-aided molecular design 1997, 11, 425-445.

(12) Nguyen, N. T.; Nguyen, T. H.; Pham, T. N. H.; Huy, N. T.; Bay, M. V.; Pham, M. Q.; Nam, P. C.; Vu, V. V.; Ngo, S. T. Autodock vina adopts more accurate binding poses but autodock4 forms better binding affinity. Journal of Chemical Information and Modeling 2019, 60, 204-211.

(13) Morris, G. M.; Goodsell, D. S.; Halliday, R. S.; Huey, R.; Hart, W. E.; Belew, R. K.; Olson, A. J. Automated docking using a Lamarckian genetic algorithm and an empirical binding free energy function. Journal of computational chemistry 1998, 19, 1639-1662.

(14) Ain, Q. U.; Aleksandrova, A.; Roessler, F. D.; Ballester, P. J. Machine-learning scoring functions to improve structure-based binding affinity prediction and virtual screening. Wiley Interdisciplinary Reviews: Computational Molecular Science 2015, 5, 405-424.

(15) Zitnik, M.; Nguyen, F.; Wang, B.; Leskovec, J.; Goldenberg, A.; Hoffman, M. M. Machine learning for integrating data in biology and medicine: Principles, practice, and opportunities. Information Fusion 2019, 50, 71-91. 
(16) Berman, H. M.; Westbrook, J.; Feng, Z.; Gilliland, G.; Bhat, T. N.; Weissig, H.; Shindyalov, I. N.; Bourne, P. E. The protein data bank. Nucleic acids research 2000, 28, 235-242.

(17) Wang, R.; Fang, X.; Lu, Y.; Wang, S. The PDBbind database: Collection of binding affinities for protein- ligand complexes with known three-dimensional structures. Journal of medicinal chemistry 2004, 47, 2977-2980.

(18) Liu, Z.; Su, M.; Han, L.; Liu, J.; Yang, Q.; Li, Y.; Wang, R. Forging the basis for developing protein-ligand interaction scoring functions. Accounts of chemical research 2017, 50, 302-309.

(19) Gilson, M. K.; Liu, T.; Baitaluk, M.; Nicola, G.; Hwang, L.; Chong, J. BindingDB in 2015: a public database for medicinal chemistry, computational chemistry and systems pharmacology. Nucleic acids research 2016, 44, D1045-D1053.

(20) Ballester, P. J.; Mitchell, J. B. A machine learning approach to predicting proteinligand binding affinity with applications to molecular docking. Bioinformatics 2010, 26, 1169-1175.

(21) Zilian, D.; Sotriffer, C. A. SFCscore RF: a random forest-based scoring function for improved affinity prediction of protein-ligand complexes. Journal of chemical information and modeling 2013, 53, 1923-1933.

(22) Ballester, P. J. Machine learning scoring functions based on random forest and support vector regression. IAPR International Conference on Pattern Recognition in Bioinformatics. 2012; pp 14-25.

(23) Li, G.-B.; Yang, L.-L.; Wang, W.-J.; Li, L.-L.; Yang, S.-Y. ID-Score: a new empirical scoring function based on a comprehensive set of descriptors related to protein-ligand interactions. Journal of chemical information and modeling 2013, 53, 592-600. 
(24) Li, L.; Wang, B.; Meroueh, S. O. Support vector regression scoring of receptor-ligand complexes for rank-ordering and virtual screening of chemical libraries. Journal of chemical information and modeling 2011, 51, 2132-2138.

(25) Durrant, J. D.; McCammon, J. A. NNScore: a neural-network-based scoring function for the characterization of protein- ligand complexes. Journal of chemical information and modeling 2010, 50, 1865-1871.

(26) Ashtawy, H. M.; Mahapatra, N. R. BgN-Score and BsN-Score: bagging and boosting based ensemble neural networks scoring functions for accurate binding affinity prediction of protein-ligand complexes. BMC bioinformatics 2015, 16, S8.

(27) Ashtawy, H. M.; Mahapatra, N. R. Task-specific scoring functions for predicting ligand binding poses and affinity and for screening enrichment. Journal of chemical information and modeling 2018, 58, 119-133.

(28) Cang, Z.; Wei, G.-W. Integration of element specific persistent homology and machine learning for protein-ligand binding affinity prediction. International journal for numerical methods in biomedical engineering 2018, 34, e2914.

(29) LeCun, Y.; Bengio, Y.; Hinton, G. Deep learning. nature 2015, 521, 436-444.

(30) Krizhevsky, A.; Sutskever, I.; Hinton, G. E. Imagenet classification with deep convolutional neural networks. Communications of the ACM 2017, 60, 84-90.

(31) Brown, T. B.; Mann, B.; Ryder, N.; Subbiah, M.; Kaplan, J.; Dhariwal, P.; Neelakantan, A.; Shyam, P.; Sastry, G.; Askell, A., et al. Language models are few-shot learners. arXiv preprint arXiv:2005.14165 2020,

(32) Wallach, I.; Dzamba, M.; Heifets, A. AtomNet: a deep convolutional neural network for bioactivity prediction in structure-based drug discovery. arXiv preprint arXiv:1510.02855 2015, 
(33) Pereira, J. C.; Caffarena, E. R.; dos Santos, C. N. Boosting docking-based virtual screening with deep learning. Journal of chemical information and modeling 2016, 56, 2495-2506.

(34) Ragoza, M.; Hochuli, J.; Idrobo, E.; Sunseri, J.; Koes, D. R. Protein-Ligand Scoring with Convolutional Neural Networks. Journal of Chemical Information and Modeling 2017, 57, 942-957.

(35) Feinberg, E. N.; Sur, D.; Wu, Z.; Husic, B. E.; Mai, H.; Li, Y.; Sun, S.; Yang, J.; Ramsundar, B.; Pande, V. S. PotentialNet for molecular property prediction. ACS central science 2018, 4, 1520-1530.

(36) Jiménez, J.; Skalic, M.; Martinez-Rosell, G.; De Fabritiis, G. K deep: Protein-ligand absolute binding affinity prediction via 3d-convolutional neural networks. Journal of chemical information and modeling 2018, 58, 287-296.

(37) Stepniewska-Dziubinska, M. M.; Zielenkiewicz, P.; Siedlecki, P. Development and evaluation of a deep learning model for protein-ligand binding affinity prediction. Bioinformatics 2018, 34, 3666-3674.

(38) Jiang, H.; Fan, M.; Wang, J.; Sarma, A.; Mohanty, S.; Dokholyan, N. V.; Mahdavi, M.; Kandemir, M. T. Guiding Conventional Protein-Ligand Docking Software with Convolutional Neural Networks. Journal of Chemical Information and Modeling 2020,

(39) Nguyen, D. D.; Gao, K.; Wang, M.; Wei, G.-W. MathDL: mathematical deep learning for D3R Grand Challenge 4. Journal of computer-aided molecular design 2020, 34, $131-147$.

(40) Masuda, T.; Ragoza, M.; Koes, D. R. Generating 3D Molecular Structures Conditional on a Receptor Binding Site with Deep Generative Models. arXiv preprint arXiv:2010.14442 2020, 
(41) Koes, D. R.; Baumgartner, M. P.; Camacho, C. J. Lessons learned in empirical scoring with smina from the CSAR 2011 benchmarking exercise. Journal of chemical information and modeling 2013, 53, 1893-1904.

(42) O’Boyle, N. M.; Banck, M.; James, C. A.; Morley, C.; Vandermeersch, T.; Hutchison, G. R. Open Babel: An open chemical toolbox. Journal of cheminformatics 2011, 3,33 .

(43) Babel, O. Open Babel: The Open Source Chemistry Toolbox.

(44) Quiroga, R.; Villarreal, M. A. Vinardo: A scoring function based on autodock vina improves scoring, docking, and virtual screening. PloS one 2016, 11, e0155183.

(45) Francoeur, P. G.; Masuda, T.; Sunseri, J.; Jia, A.; Iovanisci, R. B.; Snyder, I.; Koes, D. R. Three-Dimensional Convolutional Neural Networks and a Cross-Docked Data Set for Structure-Based Drug Design. Journal of Chemical Information and Modeling 2020, 60, 4200-4215.

(46) Jia, Y.; Shelhamer, E.; Donahue, J.; Karayev, S.; Long, J.; Girshick, R.; Guadarrama, S.; Darrell, T. Caffe: Convolutional Architecture for Fast Feature Embedding. arXiv preprint arXiv:1408.5093 2014,

(47) Hochuli, J.; Helbling, A.; Skaist, T.; Ragoza, M.; Koes, D. R. Visualizing convolutional neural network protein-ligand scoring. Journal of Molecular Graphics and Modelling 2018, 84, 96-108.

(48) Teague, S. J. Implications of protein flexibility for drug discovery. Nature Reviews Drug Discovery 2003, 2, 527-541.

(49) Zhao, Y.; Sanner, M. F. Protein-ligand docking with multiple flexible side chains. $J$ Comput Aided Mol Des 2008, 22, 673-679. 
(50) Wierbowski, S. D.; Wingert, B. M.; Zheng, J.; Camacho, C. J. Cross-docking benchmark for automated pose and ranking prediction of ligand binding. Protein Science 2020, 29, 298-305.

(51) Bakan, A.; Meireles, L. M.; Bahar, I. ProDy: protein dynamics inferred from theory and experiments. Bioinformatics 2011, 27, 1575-1577.

(52) RDKit: Open-source cheminformatics. http://www.rdkit.org, [Online; accessed 11April-2013].

(53) Schrödinger, LLC, The PyMOL Molecular Graphics System, Version 1.8. 2015.

(54) Su, M.; Yang, Q.; Du, Y.; Feng, G.; Liu, Z.; Li, Y.; Wang, R. Comparative assessment of scoring functions: the CASF-2016 update. Journal of chemical information and modeling 2018, 59, 895-913.

(55) Peter, D. hyperfine. https://github.com/sharkdp/hyperfine, 2020.

(56) Dietterich, T. G. Ensemble methods in machine learning. International workshop on multiple classifier systems. 2000; pp 1-15.

(57) Meli, R.; Anighoro, A.; Bodkin, M.; Morris, G.; Biggin, P. Learning ProteinLigand Binding Affinity with Atomic Environment Vectors. ChemRxiv 2020, ChemRxiv:13469625. 


\section{Graphical TOC Entry}

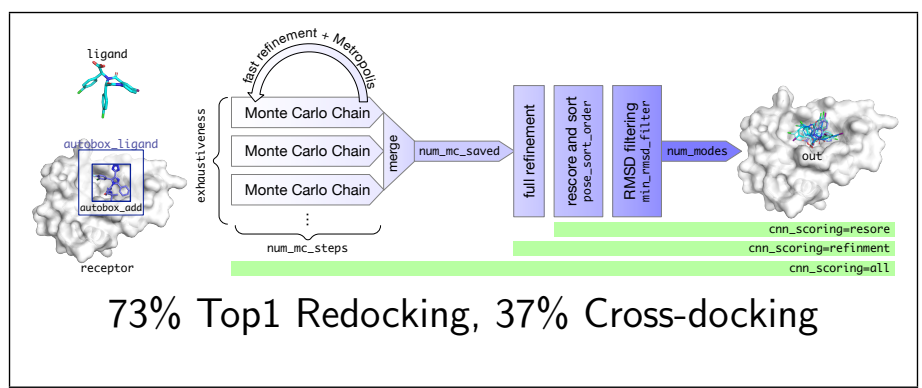

